\title{
Ciencia Ficción como Fuente de Principios Jurídicos para regular la Inteligencia Artificial*
}

\author{
Santiago Sánchez Morales**
}

\begin{abstract}
RESUMEN
El quehacer jurídico cada día va evolucionando con la introducción de nuevas tecnologías en la vida diaria de las personas. Sin embargo, la Inteligencia Artificial (IA) se vislumbra no solo como un paso más en esta evolución, sino como una transformación total en la práctica diaria del jurista. Ante esta situación, el Derecho debe reevaluar sus prácticas y sobre todo sus principios para poder adecuarse a este cambio latente y con ello no quedar obsoleto o desactualizado. Aprovechando la corriente de la Filosofía de la Experiencia Jurídica, esta evolución jurídica puede analizarse por medio del estudio de algunas obras literarias y cinematográficas de Ciencia Ficción que sirvieron como base para presentar posibles dilemas teóricos sobre la tecnología -especialmente sobre IA-que, no obstante, son actuales o probables y que obligarán a limitar, incentivar, regir o corregir, desde el Derecho, todo aquello que afecte al ser humano y por ende su esfera jurídica.
\end{abstract}

\section{PALABRAS CLAVE}

Quehacer jurídico, Inteligencia Artificial, Ciencia Ficción, Filosofia de la Experiencia Jurídica, principios jurídicos.

\begin{abstract}
The legal functions are evolving everyday with the introduction of new technologies to the people's daily lives. However, Artificial Intelligence or Al is seen not only as another step in this evolution, but as a total transformation of the daily practices of the jurist. Given this situation, the Law must reassess its practices, and specially its principles, in order to adapt to this latent change and thus not become obsolete or outdated. Taking advantage of the school of Philosophy of Juridical Experience, this legal evolution can be analise through the study of some literature and cinematographic Science Fiction' works that served as foundation to show theoretical dilemmas about technology-specially about Al- which, nevertheless are present or probable and that will force to limit, encourage, govern, or correct, through the Law, anything that may affect the human being and therefore his legal sphere.
\end{abstract}

\section{KEYWORDS}

Legal function, Artificial Intelligence, Science Fiction, Philosophy of Juridical Experience, juridical principles.

\footnotetext{
*Artículo de Investigación postulado el 11 de febrero de 2020 y aceptado el 7 de diciembre de 2020

${ }^{*}$ Coordinador de Posgrados y Coordinador de Investigación en la Facultad de Derecho de la Universidad Panamericana, Aguascalientes, México. (sasanchezm@up.edu.mx) orcid.org/0000-0002-9999-7714
} 


\section{SUMARIO}

1. Introducción

2. Relación Derecho e inteligencia artificial

3. Sobre la Filosofía de la Experiencia Jurídica

4. La necesidad de principios jurídicos

5. Un ejemplo en la Tres Leyes de la Robótica

6. Bibliografía

\section{Introducción}

Cuando hablamos de Ciencia Ficción las principales ideas que vienen a la cabeza son aquellas que refieren al cine, a la televisión y la literatura, esto sucede aún más cuando se le condiciona al término de Inteligencia Artificial o abreviadamente IA (AI por su siglas en inglés). Bien lo dice B. Buchanan

Science fiction writers have used the possibility of intelligent machines to advance the fantasy of intelligent nonhumans, as well as to make us think about our own human characteristics. Jules Verne in the nineteenth century and Isaac Asimov in the twentieth are the best known, but there have been many others. ${ }^{1}$

Sin embargo, no podemos descartar el avance científico de nuestro mundo y las implicaciones que este desarrollo conlleva. Garry Kasparov² durante el video inaugural a la Landmark Conference de la Organización para la Cooperación y Desarrollo Económicos intitulada “AI: Intelligent machines - Smart policies” dice a los diferentes actores, ponentes y asistentes "There could be no better time [...] to address the issue of intelligent machines and human policies. AI is not science fiction it's the present and will affect more aspects of our lives every day". ${ }^{3}$

No está exento de esta realidad el mundo jurídico. Los términos Informática Jurídica o Legaltech ${ }^{4}$ son prueba internacional y/o doctrinal de que la

\footnotetext{
'Buchanan, Bruce G. "A (Very) brief history of Artificial Intelligence" Al Magazine, American Association for Artificial Intelligence (AAAI), Volumen 26, No. 4, año 2006, invierno 2005 p. 53

${ }^{2}$ Garry Kasparov fue campeón del juego de ajedrez desde 1985 hasta el año 2000, y también fue uno de los principales competidores de ajedrez contra la IA "Deep Blue" la cual derrotó a Kasparov en 1997, convirtiéndose este último en el primer sistema de inteligencia artificial capaz de superar a cualquier humano en el juego.

${ }^{3}$ OECD, "Al Conference Welcome Message" [en linea]. En Going Digital. Al Intelligent machines, Smart policies. 26 octubre 2017 [fecha de consulta 20 diciembre 2019]. Disponible en: https://www.oecd.org/going-digital/ai-intelligent-machines-smart-policies/conference-agenda/

${ }^{4}$ Aunque ambos términos no son per se sinónimos, la gran mayoría de los estudiosos entienden que el término
} 
tecnología está afectando el quehacer de los servicios jurídicos. Aunque los términos son relativamente novedosos y están en boga en el lenguaje de los principales despachos jurídicos, múltiples autores y periodistas han contribuido a esparcir la muy fundamentada visión sobre el abogado del siglo XXI. Este abogado, conocido como abogado digital, se está viendo comprometido en su actividad gracias a la automatización de procesos, especialmente en aquellas labores antes referidas para los estudiantes o iniciados como pasantes, practicantes o noveles, según la terminología en cada región.

Una experiencia de lo anterior, podemos decir, está en la creación de contratos, donde una máquina es capaz de utilizar todo el conocimiento de internet, de bases de datos y demás contenido jurídico digital para crear un contrato utilizando lo mejor de diversos sistemas jurídicos, de cláusulas y de redacciones previas para satisfacer la necesidad del cliente, algo que pudiéramos decir casi sin temor a equivocarse, ningún abogado consumado es capaz de realizar mucho menos en tiempo récord, qué decir de un iniciado a quienes muchas veces estaba destinado este tipo de labores de forma tradicional. ${ }^{5}$

Dejando de lado lo general de la tecnología y entrando en materia, la manera en que el abogado comprende y utiliza el término de Derecho y sus conceptos jurídicos fundamentales se está viendo transformado por el desarrollo de la -no ficticia- inteligencia artificial, ya que la aplicación de esta última ha llevado a repensar aquellas definiciones clásicas de persona jurídica, supuesto jurídico, hecho y acto jurídico, consecuencia de derecho, sanción, coacción, derecho subjetivo y -el caso que se abordará en este trabajo- los principios jurídicos. Sirva de ejemplo temas como contratos inteligentes o autoejecutables como medio de coacción a las partes contratantes, o la responsabilidad de objetos autónomos que implican una responsabilidad no humana ante un daño a terceros, por mencionar algunos paradigmas existentes hoy en día de tecnología imbuida con IA. Por tanto, es menester abordar en este trabajo este nuevo paradigma para el quehacer del abogado.

Legaltech es la más actual de las evoluciones de la Informática Jurídica. La división se debe en razón que la Informática es la aplicación de sistemas informáticos para la facilitación del derecho mientras que Legaltech no nada más implica facilitación sino innovación, permitiendo acceder a herramientas de comercialización y competencia que ayuden tanto al abogado como al propio cliente.

${ }^{5}$ Existen estudios donde se han realizado comparaciones entre el tiempo que le toma a un abogado consumado realizar un contrato respecto del que requiere una inteligencia artificial, esto con el fin de probar la eficiencia de trabajo entre unos y otros. Los resultados en varias ocasiones han demostrado que para una eficiencia equiparable a la del humano, muchos programas de IA pueden lograr el cometido en porcentajes inmejorables de tiempo, incluso con costos mucho más accesibles para un despacho de un cierto nivel de ingreso. 
Pero, ¿Cómo lograr advertir los avances y posibles nuevos usos, sus afectaciones al mundo del derecho y en general en su desarrollo en la vida diaria? Si bien es imposible predecir cada uno de los logros técnicos de los próximos años en esta rama, el único numen real que tiene el ser humano -y en especial la Ciencia Jurídica- sobre este tipo de tecnologías se encuentra en el ideario colectivo presente en la Ciencia Ficción. Por ello esta debe ser la principal inspiración para el Derecho, así como lo fue y ha sido para la ingeniería. De igual forma, la cuestión sobre cómo aterrizar estas ideas literarias y/o cinematográficas y transformarlas en reglas, normas y expresiones jurídicas que obliguen a limitar, incentivar, regir o corregir, es trabajo de la Filosofía del Derecho y para este autor la corriente más adecuada corresponde a la Filosofía de la Experiencia Jurídica.

Por tanto, será el propósito de este trabajo alinear estas ideas con el objetivo de plantear, desde la Filosofía de la Experiencia Jurídica y la Ciencia Ficción, una forma de aproximar el Derecho a los principios necesarios para orientar jurídicamente la Inteligencia Artificial.

\section{Relación Derecho e inteligencia artificial}

Antes de iniciar el análisis del trabajo vale la pena detenerse en dos antecedentes importantes.

El primero de ellos: la relación que existe entre Derecho e Inteligencia Artificial.

Aunque parece ser una situación novedosa por su pequeños choques disciplinarios, ya el propio Buchanan -citado anteriormente- en 1970 había iniciado esta conversación junto con Thomas E. Headrick en su artículo titulado "Some speculation about artificial intelligence and legal reasoning".

Ambos autores explican brevemente el problema:

Interdisciplinary work between the lawyer and the computer scientist has floundered on the misconceptions that each has of the other's discipline. As a result, no one has yet attempted computer programs incorporating complex techniques of legal reasoning. Even efforts in legal information retrieval have been hampered by these misconceptions. In retrieval, lawyers have viewed the computer as, at most, a storehouse from which cases and statutes might be retrieved by skilfully designed indexing systems. Computer scientists, on the other hand, have operated with a conception of law as a collection of facts and "correct" legal principles; they have assumed that the computer can be most helpful 
to the lawyer if it can retrieve the right answers quickly. But the lawyer rarely looks for, or even expects, clear answers. ${ }^{6}$

Estos malentendidos -aquí ejemplificados con cuestiones de investigación jurídica- entre abogados e ingenieros a los que refieren Buchanan y Headrick han hecho que una y otra disciplina trabajen separadamente. Así pues, en lugar de permitir a la inteligencia artificial eficientar e innovar el trabajo jurídico, también se ha separado lo jurídico de la inteligencia artificial. Si bien, esta afirmación anterior pudo ser considerada generalmente cierta en un inicio y actualmente la relación entre Derecho e Inteligencia artificial no se puede considerar estrecha, si tiene ciertos y determinados campos donde han trabajado de la mano haciendo que con el paso del tiempo sea más notable su interoperabilidad para el beneficio de ambas disciplinas.

Este es el caso del propio lenguaje jurídico, donde términos como "código" (como algoritmo computacional) o "dominio" (como nombre de alojamiento de una página web) son parte de la "jerga” jurídica que tienen un significado jurídico pero también tecnológico. Y ni se diga aquellos términos del lenguaje técnico han pasado al jurídico como es e-commerce, activos virtuales o los datos personales y que ahora son parte de la dinámica diaria del derecho.

Es necesario hacer un pequeño paréntesis en la explicación para tener una mayor esquematización de los campos arriba señalados: Es decir, la distinción entre IA aplicada al derecho -que es esencialmente el campo de estudio de los sistemas técnicos al trabajo jurídico- y el derecho de la IA -que refiere principalmente a la regulación de la técnica en su aplicación cotidiana incluida su aplicación en el derecho-. La primera de estas distinciones ha sido el objeto de estudio de la informática jurídica como expresa Casanovas

Con el nacimiento de las grandes bases jurídicas de datos, privadas o públicas, en los años setenta y ochenta, el interés por los sistemas de clasificación en bases relacionales se incrementó. Los precedentes son muchos y distintos en cada país. En los años sesenta, este campo se denominó jurimetría. En los años setenta y ochenta empezó a ser conocido como informática jurídica. Las técnicas de IA han ocupado desde entonces una posición central en su desarrollo, como recogen también los manuales más recientes. ${ }^{7}$

\footnotetext{
${ }^{6}$ Buchanan, Bruce G. \& Headrick, Thomas E. "Some speculation about artificial intelligence and legal reasoning" Stanford Law Review, Volumen 23, No. 1, año 1970, noviembre p. 40

${ }^{7}$ Casanovas, Pompeu "Inteligencia Artificial y Derecho: a vuelapluma" Teoría y derecho: revista de pensamiento
} 
De igual forma es un área con muchos campos de acción, algunos de estos los menciona Richard Susskind ${ }^{8}$ al hablar de los tipos generales de sistemas expertos aplicados al derecho, otros como Mateo Borge ${ }^{9}$ expresan campos más cerrados y con aplicación específica, aunque son clasificaciones que vale la pena tener en cuenta si se busca estudiar más a fondo esta rama, que, sin embargo, no es propósito de este trabajo. No obstante, es importante señalar que estos campos, junto con su desarrollo, también reflejan un tipo de implementación en la actividad jurídica. "Poco a poco, los despachos de abogados están empezando a coquetear con las herramientas características de la nueva revolución industrial, aunque un bufete 4.0 todavía es algo que no puede encontrarse en el mercado." ${ }^{10}$

Por otro lado, la regulación de la Inteligencia Artificial es un campo como muchas autores han descrito, que "supone la entrada en un mundo totalmente desconocido, con riesgos impredecibles, que provoca ab initio una palpable inseguridad jurídica debida sobre todo a la inexistencia de un marco regulador claro. $^{11}$

Esta falta de claridad se debe propiamente al poco entendimiento sobre lo que es la inteligencia artificial. La profesora Alzbeta Krausova lo expresa muy bien al describir que es un concepto ambiguo por la relatividad de su perspectiva legal siendo que esta nace de la misma esencia de lo que significa inteligencia. Ante este panorama, el derecho ha limitado su relación y su regulación principalmente a su consideración como un software, o programa computacional, por tanto, susceptible de protección por medio de la Propiedad Intelectual. De igual forma, al ser un producto de mercado ya sea como software (ej. Siri o Alexa, asistentes personales de Apple y Amazon) o imbuido en una pieza de robótica (ej. SCORP, o los perros robot guía), deben estar sujetos a ciertos estándares industriales que pueden ser contemplados en Normas Oficiales o en su genérico internacional ISO. ${ }^{12}$

jurídico. No. 7, año 2010, p. 210, Versión del autor disponible en Researchgate.net

${ }^{8}$ El trabajo de Susskind lleva por nombre "Artificial Intelligence, Expert systems and Law" Denning Law Journal No. 5, año 1990, p. 105-116 aunque esta clasificación puede ser encontrada en múltiples obras diversas tanto del autor, como de estudiosos de la informática jurídica.

${ }^{9}$ De estos autores vale la pena recalcar sus capitulos dentro del libro: Inteligencia Artificial, Tecnología y Derecho, España, Tirant Lo Blanch, 2017, ISBN 978-84-9169-720-6, sin embargo gran parte del Departamento de Derecho Civil de la Universidad de Barcelona tienen obras que refieren a estos temas y que pueden ser considerados referentes de actualización.

${ }^{10}$ Borge, Iván Mateo. "La robótica y la inteligencia artificial en la prestación de servicios jurídicos" En Inteligencia Artificial, Tecnología y Derecho, España, Tirant Lo Blanch, 2017, ISBN 978-84-9169-720-6 p. 128

"Ibidem p. 145

${ }^{12}$ Krausova, Alzbeta. "Intersections between Law and Artificial Intelligence", International Journal of Computer, (IJC), Volumen 27, No. 1, año 2017 
Otras cuestiones que se han visto puestas en la lupa del derecho son las relacionadas con la privacidad y los datos personales que, en últimos años y a causa de escándalos mediáticos, han tenido un acercamiento parcial al problema dando como resultado Leyes y reglamentos sobre Protección de datos personales, pero donde la IA puede ser considerada, solo como un medio de obtención de los mismos, no como una herramienta que necesita de información para trabajar lo que implica un sinfín de cuestionamientos que se abordarán más adelante.

Finalmente aplicaciones ya existentes como en la banca (ej. La compraventa automática de acciones sin intervención humana, o las criptomonedas), la defensa (como los drones no tripulados, o las ahora en boga Lethal Autonomous Weapon Systems o LAWS) o las salud (ej. Sistemas de detección predictiva de enfermedades) son algunos usos específicos que tanto a nivel internacional como nacionales tienen algún tipo de regulación pero que han sido necesarias para resolver una realidad sobrepasada y que no necesariamente corresponden a una solución a futuro. Mucho como el dicho mexicano “Muerto el niño, a tapar el pozo".

\section{Sobre la Filosofía de la Experiencia Jurídica}

Después de vislumbrar la relación tan limitada que existe entre Derecho e Inteligencia artificial, como segundo antecedente es importante exponer en breve la corriente de la Filosofía de la Experiencia Jurídica puesto que es la base iusfilosófica y metodológica para continuar con este estudio. Paralelamente, dado que no es una corriente conocida en América latina por ser enarbolada principalmente por autores italianos -como son Giuseppe Capograssi o Sergio Cotta- con alguno que otro español -como Jesús Ballesteros o Fernando H. Llano- tratarlo como un antecedente de contexto resulta todavía más importante.

Estos autores definen la "Experiencia Jurídica" como una amalgama, "como una perplejidad que causa en quienes lo estudian, la enorme variedad de enfoques e interpretaciones doctrinales que del mismo podrían hacerse" ${ }^{13}$, lo que nos obliga a decantarnos por una postura, la cual corresponderá a aquella que expone Fernando H. Llano en su Libro El formalismo jurídico y la Teoría Experiencial del Derecho. Dado que el autor al hacer un recuento de todos estos diferentes derroteros decide sintetizar la idea de Experiencia Jurídica como "al

\footnotetext{
${ }^{13}$ Llano Alonso, Fernando H. El formalismo jurídico y la Teoria Experiencial del Derecho. España. Tirant Lo Blanch, 2009 ISBN 978-84-9985-511-0, p. 50
} 
menos [...] una reflexión crítica, explicativa y omnicomprensiva de la realidad que está ligada a la problemática inherente a la propia existencia del Derecho como fenómeno" ${ }^{14}$ entonces -la Filosofía de la Experiencia Jurídica- no puede ser considerada una escuela ni una doctrina institucionalista, realista o sociológica ya que su leitmotiv responde a "una visión del Derecho que abarque su entero desenvolvimiento desde su génesis en la conciencia individual, para a través de su plasmación consuetudinaria y legislativa, su individualización judicial, arribar a su elaboración crítica por la doctrina”. ${ }^{15}$

Así podemos simplificar que la experiencia jurídica es una forma no solo de estudiar y conocer el derecho, sino que implica vivir el derecho, concebirlo como un fenómeno con un devenir propio que nace en las ideas del ser humano. Estas ideas no son espontáneas, sino que como todo lo humano, tienen un peso en la realidad, en la crítica, en la explicación, en la imaginación de éste, las cuales serán plasmadas de forma práctica en los usos sociales, culturales o también tomadas por un legislador, juzgador, jefe de estado o incluso un superior cualquiera (no necesariamente de gobierno) y ser impuestas como normas, principios, o resoluciones que afecten y transformen la vida social, económica, cultural según su nivel de influencia.

Por tanto, este modo de vida del Derecho obliga al estudio fenomenológico de la realidad que permite al ser humano inspirar su idea del derecho; que permite utilizar todos los medios usados -sus fuentes- para conformar, comprender y exponer aquello que transformará y orientará a la realidad social, cultural, económica, política en la que la persona convive y participa. ${ }^{16}$

Sergio Cotta expresa con mucha elocuencia esta expresión, tanto así, que el propio Fernando Llano retoma esta idea en su libro citado anteriormente: "El conocimiento del objeto del Derecho y el conocimiento del sujeto viviente de modo jurídico se implican recíprocamente y esta coimplicación de ambos es la que nos permitirá llegar a la comprensión del sentido del Derecho" ${ }^{17}$ Sentido que nos permitirá en el propósito de este trabajo no solo conocer la necesidad del Derecho y su transformación con la Inteligencia artificial en el caso

\footnotetext{
${ }^{14}$ Ibidem, p. 111

${ }^{15}$ Cita de Perez Luño en Llano Alonso, Fernando H. El formalismo... op. cit., p. 112 En otras palabras, la Filosofía de la Experiencia Jurídica no está catalogada como un pensamiento uniforme sino como una forma de concebir el Derecho desde cualquiera de las escuelas y doctrinas que comprenden al Derecho como una Ciencia Práctica.

${ }^{16}$ El término "vivir el derecho" es muy usado por la Filosofía de la Experiencia Jurídica con el fin de ejemplificar esta dimensión práctica y fenomenológica. Sabiendo que el Derecho nace de la sociedad, por tanto, se desarrolla en la sociedad y para la sociedad como un fenómeno más el cual tiene inspiraciones y necesidades que nacen de lo propio del ser humano.

${ }^{17}$ Llano Alonso, Fernando H. El formalismo... op. cit., p. 220
} 
específico, sino también -conocer y- cómo utilizar las fuentes de inspiración que nos permitan vivir la experiencia jurídica que es la Inteligencia Artificial.

Tal afirmación permite comenzar a responder la pregunta que plantea este antecedente: ¿Cómo puede estudiar esta corriente filosófica la Inteligencia Artificial? Hagamos reingeniería inversa para expandir y ligar una con otra a expensas que podamos obtener una observación más adelante.

Si decimos que la Inteligencia Artificial es susceptible de ser pensada, criticada, o comprendida de forma racional -tanto así que es una disciplina de la ingeniería computacional-y que este estudio tiene o puede tener incidencia en la realidad -mejor llamada tecnología-, por tanto, tiene una consecuencia que repercute de alguna forma en el Derecho. Lo anterior significa entonces que estamos ante un fenómeno que se vive en la sociedad o la cultura con múltiples expresiones, una de las cuales necesariamente es legal, por tanto, según nuestra definición anterior es definible la IA como una Experiencia Jurídica.

Otra manera de ejemplificar ello está dicho por Granfield en La experiencia interna del Derecho, que para conocer el sentido del Derecho en un inicio se necesita tener datos proporcionados por los sentidos además de conciencia y luego una interacción con otro sujeto. ${ }^{18}$ En un primer acercamiento esto no tendría una relación, sin embargo si entendemos que esta conciencia y los sentidos son otro modo de concebir la Experiencia Jurídica, entonces estamos ante una metodología de creación de experiencia que nos es más palpable a la normalidad humana. Este acercamiento por los sentidos y la conciencia que tenemos de ella es manera de reelaborar lo mismo que hemos dicho, pero que nos permite agregar un nuevo derrotero y que es de total relevancia para el trabajo.

Una aseveración que podemos realizar casi indefectiblemente, sobre la conciencia de la población y cómo se percibe en lo cotidiano la Inteligencia Artificial, es que gran parte de las ideas que se han realizado en esta área nacen de la tecnología existente actualmente, sistemas como Alexa de Amazon o Siri de Apple. Pero que, como sabemos nacen de ideas preconcebidas en la ciencia ficción que "puede ser definida como aquella rama de la literatura [e incluyamos aquí también al cine] que trata sobre las reacciones de los seres humanos a los cambios en la ciencia y la tecnología."19

Sin embargo estas reacciones no nada más han servido como "intención deliberada de predecir aquello que ha de ocurrir realmente" ${ }^{20}$, dijo Asimov, sino

\footnotetext{
${ }^{18}$ Granfield, David. La experiencia interna del derecho. Una jurisprudencia de la subjetividad. México, Catholic University of America / Universidad Iberoamericana, 1996, ISBN 968-859-228-5 p. 89

${ }^{19}$ Asimov, Isaac. Asimov on Science Fiction. EUA, Harper Collins Publishers, 1983, ISBN 978-02-4612-0441 p. 59 ${ }^{20}$ lbid
} 
como una avenida tanto de creatividad como de expresión [que ha resultado en que] la literatura y los medios de Sci-Fi han impulsado avances en tecnología interactiva y han probado ser una fuente clave de inspiración para los investigadores en el campo de la tecnología computacional $^{21}$

Lo que quiere decir, para ponerlo en el lenguaje que se ha estado usando, que la Ciencia Ficción en vez de ser una expresión de la experiencia del avance tecnológico, se ha convertido en la fuente de experiencia para la creación de ese desarrollo. Se ha transformado en el medio de divulgación, de concientización y de captación de las ideas sobre tecnología y por ende, el conducto para que tanto investigadores como público en general vivan esta nueva realidad fabricada.

Teniendo en cuenta todo esto, podemos concluir que si la Inteligencia artificial -como disciplina- es una experiencia que nace en su inspiración o tiene mucho de su fuente en la Ciencia Ficción, entonces el estudio de ésta, desde un punto de vista iusfilosófico experiencial, puede -y debiera- ser fundamento para que el Derecho cumpla su sentido ante la realidad vivida hoy en día.

\section{La necesidad de principios jurídicos}

Siguiendo la ruta discursiva sobre cómo el Derecho puede tener un mejor acercamiento al fenómeno de la Inteligencia Artificial, surge la necesidad de hablar de la que para este autor es su mejor herramienta: Los principios jurídicos.

Al hablar de Principios Jurídicos es imprescindible hablar un poco de la noción expuesta por el profesor de la Universidad de Oxford, Ronald Dworkin quien fue el principal impulsor de esta Teoría del Derecho como una forma de superar el positivismo instaurado por Kelsen y puesto, quizá, en su máxima expresión por H.L.A Hart.

Para el sucesor de Hart, en la Cátedra de Filosofía del Derecho (Jurisprudence en inglés), los principios jurídicos son

estándares, que no son normas, y que han de ser observados, no porque favorezcan o aseguren una situación económica, política o social que

\footnotetext{
${ }^{21}$ Jordan, Philipp; Mubin, Omar; Obaid, Mohammad \& Sila, Paula A. "Exploring the referral and Usage of ScienceFiction in HCl Literature". En Marcus, Aaron \& Wang, Wentao (Eds.) Design, user experience, and usability: Designing Intearctions, Tomo II, EUA, Springer, ISBN 978-3319918020 p. 19
} 
se considera deseable, sino porque es una exigencia de la justicia, la equidad o alguna otra dimensión de la moralidad. ${ }^{22}$

El origen de los principios, por tanto, escapa de la convencionalidad del sistema positivo o de lo considerado como norma y lo tiene en la ambigüedad que presenta resolver el caso concreto -cada quién lo que le corresponde reza aquella definición de Ulpiano sobre el Derecho y la Justicia-. Así también, su validez depende indefectiblemente del contenido que conllevan y sirven como directriz en la toma de decisiones más que como un supuesto específico con una carga económica y política definida. ${ }^{23}$

Con el paso del tiempo su propia ambigüedad ha llevado a entender que existen dos sentidos en cómo comprender la idea de principios jurídicos. Los principios denominados como "fuertes" son aquellos estándares cuyo contenido está inmersamente relacionado con "aquello primero desde o a partir de lo cual algo es, se hace o se conoce" ${ }^{24}$ como cita Vigo de la Metafísica de Aristóteles, es decir son los derechos que están ligados a la constitución, identificación o reconocimiento de algo como jurídico y cuyo principal enfoque actual se da en los derechos humanos. Debido a esto, son una realidad que, aún a pesar de la norma, son reconocidos como derecho y deben ser observados como prioridad o fundamento de toda decisión tanto jurisdiccional, como administrativa o inclusive cualquier decisión particular y/o personalmente jurídica.

Por otro lado, están aquellos principios jurídicos denominados "débiles” que refieren más a "un papel de 'principios' respecto a otras normas o a otros principios más débiles en virtud de haberlo dispuesto así alguna decisión autoritativa" o en su caso, "constituidos como tales a consecuencia de algún acto humano con capacidad para ello" 25 . Esta decisión autoritativa o capacidad del

\footnotetext{
${ }^{22}$ Cita de Ronald Dworkin en Vigo, Rodolfo L. Interpretación Jurídica: del modelo iuspositivista legalista decimonónico a las nuevas perspectivas. Argentina. Rubinzal. 1999, ISBN 978-950-727-197-7, p. 132 El capítulo del libro de donde se extrae llamado "Teoría distintiva "fuerte" entre normas y principios jurídicos" puede buscarse en su versión original dentro del libro coordinado por Massini, Carlos I. El iusnaturalismo actual. Argentina, Abeledo-Perrot, 1996, ISBN 950-20-0957-6

${ }^{23}$ Dworkin y sus seguidores desarrollaron la noción de Principio Juridico, bajo la base de ser una fuente del derecho aplicable de forma abstracta sustentada en valores externos al derecho positivo. Es decir, como un estándar que debiera seguir el derecho para buscar la justicia en el caso concreto, recayendo su validez en el propio contenido ético que rige la relación humana y no en el interés político, social, económico y normativo del derecho positivo.

${ }^{24}$ Cita de Aristóteles Vigo, Rodolfo L. "Los principios jurídicos y su impacto en la teoría actual." En Vigo, Rodolfo L. La interpretación (argumentación) jurídica en el Estado de Derecho Constitucional. México, Tirant Lo Blanch, 2017, ISBN 978-84-9169-616-2 p. 144

${ }^{25}$ Ibidem, p. 145
} 
acto humano no es exclusiva de una autoridad sino que también apela a cualquier relación jurídica que requiera ese elemento de justicia, moralidad, etc.

En definitiva, ambos enfoques pueden ser considerados como un esquema de regulación no normativa, que viene derivado de un contenido sea fundamental, sea decisorio o una actividad humana con consideración jurídica y que conlleva la función de "fundamentar el ordenamiento jurídico, orientar en la operatividad al derecho vigente y ser fuente supletoria o integradora del ordenamiento" 26

Sin embargo ¿Qué hace de los principios la mejor herramienta jurídica para relacionar el Derecho con los apartados anteriores sobre inteligencia artificial, experiencia jurídica y lo expuesto en un inicio como quehacer jurídico y Ciencia Ficción? Respondamos esta cuestión en partes.

La primera parte refiere a la eminente practicidad que implica cada uno de los conceptos de forma común. El objeto de estudio a nivel jurídico que representa la IA no refiere a un objeto conceptual, abstracto o etéreo, sino a una disciplina de la actividad humana (específicamente de la invención humana) que tiene en su nivel técnico un sinfín de aplicaciones, usos y formas, todas relacionadas a la capacidad de aplicar algún tipo de concepción de inteligencia. En segundo término, como ya se dijo, la experiencia jurídica implica la vivencia factual del derecho, es decir la experiencia práctica del derecho y su estudio conducente, mientras que no podemos descartar al mismo tiempo la definición extendida del Derecho como parte de los saberes prácticos:

Decimos que es práctico, porque dicho saber se compromete con el campo de las conductas y sus reglas a las que orienta, legitima o descalifica con argumentos racionales. A diferencia de los saberes teóricos propios de los espectadores, el saber jurídico corresponde a los protagonistas en tanto lo que se sabe requiere ciertos comportamientos y desecha otros. ${ }^{27}$

Estos protagonistas, que son los juristas, implican que su quehacer es eminentemente práctico. Así en un tercer momento, podemos incluir que los principios jurídicos comparten esta naturaleza puesto que son parte de esas reglas argumentadas de forma racional con un origen en un comportamiento o contenido que parte de la praxis humana y que sirve de fundamento, orientación o fuente para la ciencia jurídica.

\footnotetext{
${ }^{26}$ Vigo, Rodolfo L. Interpretación Jurídica: del modelo... op. cit. p. 139

${ }^{27}$ Ibidem, p. 155
} 
Finalmente la Ciencia Ficción, como ya se dijo, es la principal fuente de inspiración en todos sus formatos -literarios, cinematográficos, etc.- para que la disciplina de la Inteligencia Artificial tenga un plausible rumbo en nuestro ambiente, así como la principal exhortación a la realidad tecnológica a la que nos encaminamos con el desarrollo científico día a día, como se citaba a Kasparov al inicio de este trabajo: "AI is not science fiction it's the present and will affect more aspects of our lives every day". ${ }^{28}$

Una segunda etapa corresponde a su contextualidad. Cada uno de estos conceptos, requiere ser comprendido desde un contexto, pero ante todo debe ser aplicado a un contexto. La experiencia jurídica, así como el quehacer jurídico casi son sinónimos de esta idea -por un lado, la vivencia contextual del derecho y la aplicación al caso concreto por el otro-, puesto que se requiere comprender el ambiente donde se desenvuelven necesariamente para poder realizar una valoración racional y en el primer caso poder desentrañar la idea jurídica del hecho estudiado, en el segundo poder darle el sentido justo, equitativo, de orden al hecho específico presentado.

Lo mismo sucede con los principios, los cuales, aunque se expresan como abstractos, solo pueden ser aplicados según el orden que se presenta en el momento en que se necesitan, un ejemplo muy común puede ser el principio retroactividad que solo puede aplicarse si en el momento específico no se violenta la seguridad jurídica de un individuo o sus derechos adquiridos.

Asimismo, los principios también comparten esta etapa con la Ciencia Ficción. En un inicio y ante una lectura o vista ligera, esta expresión mediática tiene su propio contexto ya sea futurista, utópica, espacial, dimensional, como sea. Sin embargo, sus autores no buscan apelar a que su lectura se dé en esos tiempos ni dimensiones, sino al contexto actual del lector específicamente, por tanto, existen elementos dentro de ella que hacen sentir una familiaridad con los elementos y eventos.

Esto significa a menudo una extrapolación del presente, una extrapolación que es tan clara y obvia como pronosticar algo que es inevitable. Cuando esto ocurre, el escritor de ciencia ficción ha hecho efectivamente una predicción exitosa.

Habitualmente esto sorprende a casi todo el mundo, porque aún hoy en día la humanidad da por sentado que las cosas no cambian. ${ }^{29}$

\footnotetext{
${ }^{28} 2017$ OECD Al Conference Welcome Message [en línea]... op. cit.

${ }^{29}$ Asimov, Isaac. Asimov on Science... op. cit. p. 59
} 
De esta forma su contexto se vuelve el nuestro, sus ideas nos apelan y nos inspiran emociones, ideas, miedos, se convierten para los viewers parte de su vivencia experiencial de la tecnología lo que lleva a que sean parte de su vida diaria.

En última instancia dejamos a la Inteligencia Artificial, más por una cuestión de complejidad que porque se deslinde de etapa presente. La tecnología como desarrollo científico siempre tiene un sentido contextual, ya que en su mayoría busca resolver una problemática o necesidad específica, es en sí, una solución técnica al caso concreto. No obstante como la Inteligencia Artificial puede ser aplicada a múltiples casos y puede tener múltiples aplicaciones parecería que su contextualidad es tan inagotable que no sería posible su ordenación, o en términos jurídicos su regulación. Esto sería cierto si únicamente se atendiera esta característica, sin embargo al confluir ésta con la practicidad vemos que el campo se reduce considerablemente lo que permite realmente su valoración jurídica puesto que resultan en experiencias comunes.

Pongamos un ejemplo general y uno específico, que además sirva de adelanto del propósito de las etapas. En el universo de Star Wars conocemos a un robot llamado $\mathrm{C}-3 \mathrm{PO}$, este personaje siempre se introduce bajo el mote " $\mathrm{Hu}$ man-Cyborg relations" en español "Relaciones cibernético-humanas" y tiene la capacidad de traducir seis millones de idiomas, lo que le permite lograr su propósito de generar relación entre diferentes humanos y máquinas, sin embargo, este propósito solo se lograría si el propio androide tuviera la capacidad de entender el contexto de los idiomas y no nada más una traducción literal. Así, la funcionalidad de un androide como $\mathrm{C}-3 \mathrm{PO}$ requiere también de una enorme base de datos que permita contextualizar sus protocolos. En nuestra realidad, el ejemplo de descontextualización de una IA lo tenemos con Google Translate, donde la traducción del servicio ha sido objeto de burla por errores de esta índole y que con el paso del tiempo y el nutrido de datos, ha hecho que esta aplicación sea cada vez más eficiente.

Lo mismo sucede cuando extrapolamos no a idiomas, sino a interacciones, la funcionalidad de un robot o de una IA depende necesariamente de la capacidad de contextualizar las necesidades del cliente para obtener un resultado lo más acorde posible. Lo que conlleva a que el contexto de datos que requiere es necesariamente proporcional al tipo de funciones que debe realizar. Es decir, su practicidad depende del contexto de los datos que tenga programados y clasificar su forma de ordenación por el tipo de datos que requiere. Esta ordenación sí es objeto del Derecho, tanto que tenemos como ejemplo la Protección de Datos Personales, donde se ha visto a la IA como medio de obtención de datos. Ahora, si se evalúa detenidamente que estos datos son la herramienta 
para el funcionamiento de esta rama de la ingeniería computacional, entonces estamos ante un dilema de cómo afecta o influye el contexto de los datos en la practicidad de la máquina.

El principio de No Discriminación o de Igualdad al que se refieren la mayoría de las Instrumentos internacionales como la Carta de Naciones Unidas en su Artículo $7^{30}$ hacen referencia a la no distinción del ser humano bajo ninguna circunstancia pero ¿qué sucede cuando es una máquina la que hace la decisión según los datos presentes en su protocolo que refieren a un contexto específico? El principio entonces, ante esta frontera tecnológica adquiere una nueva dimensión, ya que no es nada más aplicable al Poder Público, o a la población en general sino también a la decisión de la IA, por lo que se vuelve labor del Derecho resolver las disyuntivas que de ella surjan.

En resumidas cuentas, al aplicar la contextualidad y la practicidad dentro de la aplicación de la IA a la vida diaria, como características que comparten todos estos elementos, nos damos cuenta que los principios comienzan a verse transformados por ésta, lo que obliga a una nueva interpretación que les permita continuar su función gracias a hacer inferencias y resolver ambigüedades ante los casos concretos que se presentan.

Finalmente, una tercera etapa refiere al carácter progresivo. Es indiscutible decir que la tecnología tiene un desarrollo cada vez más acelerado. La disciplina de la Inteligencia Artificial no es la excepción a este punto, pero tampoco lo es el Derecho y su sentido, si afirmamos que como la experiencia no es estática entonces tampoco lo es la ciencia jurídica y mucho menos la labor del jurista que evoluciona de acuerdo con estos factores anteriores.

Digamos que la Ciencia Ficción también por su propio avance histórico ha tenido un progreso, aunque esta característica remite más bien, a la anticipación de su avance científico ya sea por predicción como dijo Asimov o por inspiración como lo hemos expresado. En este medio, la ciencia ha tenido un avance que ha traído sus consecuencias buenas y malas para el mundo ficción que la rodea, y que, sin embargo, también están alineadas a los sucesos que ocurren o que se planean en nuestro presente.

Por ello, si el estudio tecnológico en gran medida busca en parte o en todo el progreso planteado por este medio, entonces parte del objetivo del derecho

\footnotetext{
30 "Todos son iguales ante la ley y tienen, sin distinción, derecho a igual protección de la ley. Todos tienen derecho a igual protección contra toda discriminación que infrinja esta Declaración y contra toda provocación a tal discriminación" Asamblea General de las Naciones Unidas. Declaración Universal de los Derechos Humanos. 217 A (III) Francia, 10 diciembre 1948 [fecha de consulta 24 enero 2020]. Disponible en: https://www.un.org/es/universal-declaration-human-rights/
} 
sería también prever las consecuencias que este progreso supone. Valga dar un ejemplo muy común y que empieza a ser debate internacional gracias al desarrollo de las Lethal Autonomous Weapon Systems o LAWS.

Uno de los principales miedos públicos de la Ciencia Ficción es que las máquinas lleguen a un estado de conciencia y avance tecnológico tal que subyuguen a la humanidad coronándose como superiores al ser humano. ${ }^{31}$ Para esto, las máquinas deben tener por lo general tres características que vemos comúnmente tanto en la literatura como en el cine e incluso en los videojuegos: a) autonomía total del ser humano de creación, operación y decisión; b) conciencia en su actuar en mayor o menor medida y; c) ver a la humanidad como un daño al mundo o como un enemigo a vencer. Es decir la IA tiene que ser capaz de "ser" completamente sin el humano. ${ }^{32}$

En cuestión de defensa resalta la autonomía de los sistemas como drones, robots, nanotecnología, que permite evitar las bajas humanas o lograr con mayor precisión los ataques armados. Esto quiere decir que la inversión en la Inteligencia artificial de defensa va muy encaminada a aquellos prototipos de Ciencia Ficción previos a su "Revolución" puesto que no buscan que "sea" sino que "haga” autónomamente. Y si tecnológicamente ese es el camino que está tomando la tecnología, ¿no debería el derecho seguirle la pista sabiendo, por la Sci-Fi, que algunas decisiones pueden ser un verdadero perjuicio para el ser humano? Con esto, en opinión de este autor, no significa que no exista una oposición internacional a su creación gracias a los principios de Paz y No Violencia, sino que la falta de perspectiva del Derecho ha logrado que su desarrollo ya sea amplio, sin reglas ni justificaciones claras regresando a la idea que se había planteado del dicho mexicano.

Siguiendo entonces, estas tres etapas planteadas de practicidad, contextualidad y progresividad, como características que tiene la interdisciplinariedad del Derecho ante la experiencia jurídica que se le presenta con el avance de la Inteligencia Artificial, observamos que los principios del derecho entonces también tienen algo de progresivo gracias a su interpretación. Sin embargo, los principios también tienen un núcleo inamovible que permite su duración a lo largo del tiempo y por encima de cualquier ordenamiento jurídico. El propio

\footnotetext{
${ }^{31}$ Este miedo generalizado lleva el nombre de "complejo de Frankenstein" en honor a la novela homónima de Mary Shelly.

${ }^{32}$ Algunos de los ejemplos más famosos los encontramos el cine: 2001 Una odisea al Espacio de Stanley Kubrick, Terminator de James Cameron, Blade Runner de Ridley Scott o Matrix de los hemanos Wachowski. En la Literatura: Frankestein de Mary Shelly. En otros medios como videojuegos: NieR: Automata de Platinum Games y Square Enix, Horizon Zero Dawn de Guerrilla Games o Portal de Valve Corporation y Electronic Arts.
} 
Vigo en una relectura de Dworkin dicta que lo inamovible de estos recae en que su origen no está sujeto a la disposición de una autoridad, sino a aquellas "exigencia[s] de la justicia, la equidad o alguna otra dimensión de la moralidad"33, y que su propósito de dirigir o brindar una razón al jurista es suficiente para su validez y continuidad en el Derecho en tanto que "permanecen formando parte del mismo mientras se les estime convenientes o justos en la determinación de derechos y deberes" ${ }^{34}$ o mejor dicho, que sigan cumpliendo su triple función de fundamentadores, orientadores y como fuente integradora.

Función que se vislumbra necesaria ante el panorama sobrepasado de lo que representa la Inteligencia Artificial como experiencia jurídica y cuya inspiración de interpretación debería provenir de la lectura iusfilosófica de la Ciencia Ficción.

\section{Un ejemplo en la Tres Leyes de la Robótica}

A manera de conclusión de este trabajo, pongamos a prueba esta relación entre elementos con uno de los emblemas de la Ciencia Ficción: las famosas Tres Leyes de la Robótica expresadas por Isaac Asimov en su relato Runaround de 1942.35

El relato inserto dentro del libro Yo, robot, sirve como médula de la relación que existe entre la sociedad humana y la robótica dentro del contexto avanzado de una sociedad humana que está en la etapa de exploración espacial. Específicamente Runaround se contextualiza durante la exploración al planeta Mercurio de dos personajes y un robot, con el objetivo de evaluar la reapertura de una Estación Minera en ese planeta diez años después de su cierre.

De igual manera, el texto describe la situación que enfrentan estos científicos ante un comportamiento anormal del robot llamado Speedy que servía a ellos como instrumento esencial de recolección puesto que su constitución mecánica estaba diseñada para soportar las altas temperaturas de la superficie mercuriana.

Hasta este momento, el contexto general y la relación con la actualidad en que se vive hoy en día tienen poco o nada de común, incluso menos si se

\footnotetext{
${ }^{33}$ Vigo, Rodolfo L. Interpretación Juridica: del modelo... op. cit. p. 134

${ }^{34}$ Ibidem, p. 136

${ }^{35}$ Para este estudio se ha usado a modo de citación la versión en español del relato distribuida por el Instituto de Formación Tecnológica, gracias a que está disponible en el siguiente sitio: http://inteligenciaeducativa.net/descargas/runaround.pdf. Sin embargo, las versiones utilizadas para formular los pensamientos aqui plasmados son Asimov, Isaac. Yo, Robot. México, Editorial Sudamericana, 2004 ISBN 978-97-0780-247-6 y Asimov, Isaac. I, robot. EUA, Penguin Random House, 2008, ISBN 978-0-553-38256-3 dado que se tuvo la oportunidad de leerlos y conversarlos pero no la posibilidad de usarlos para realizar las citas correspondientes.
} 
anticipa que para Asimov este relato ocurría en 2015, cinco años antes de la fecha de este estudio.

No obstante, un pequeño detalle sale a relucir al momento de su relectura: "Tecnológicamente hablando, incluso diez años significan mucho. [...] Pero el adelanto en robótica de aquellos días era tremendo." ${ }^{36}$ Efectivamente, esta es una afirmación aplicable de forma general a nuestra realidad y en específico si sustituimos robótica por Inteligencia Artificial a nuestro caso. Incluso una coincidencia así está muy lejos de ser una justificación del carácter que pretende este estudio.

Por tanto dejemos de lado el contexto general del relato y centremos las siguientes líneas en este emblema que representan las Tres Leyes. El propio Asimov desde la conversación de los personajes enseña cuáles son estas tres leyes:

Tenemos: Una, un robot no puede hacer daño a un ser humano, o por medio de la inacción, permitir que un ser humano sea lesionado [...] Dos, un robot debe obedecer las órdenes recibidas por los seres humanos excepto si estas órdenes entrasen en conflicto con la Primera Ley. [...] Y tres, un robot debe proteger su propia existencia en la medida en que esta protección no sea incompatible con la Primer o la Segunda Ley. ${ }^{37}$

A nivel técnico, según la conversación del texto existe una distinción entre el peso que tiene para la máquina cada una de estas leyes, empezando desde la última hasta la primera.

El conflicto entre las varias reglas es allanado por los diferentes potenciales positrónicos del cerebro. Digamos que un robot se está dirigiendo a un peligro y lo sabe. El potencial automático que establece la Regla 3 le hace retroceder. Pero imagínate que le ordenas que vaya a ese peligro. En este caso, la Regla 2 establece un contrapotencial mayor que el anterior y el robot sigue las órdenes arriesgando la existencia. [...] De acuerdo con la Regla 1, un robot no puede ver cómo a un humano le sucede algo malo [...] La dos y la tres no pueden nada ante ello. ${ }^{38}$

\footnotetext{
${ }^{36}$ Asimov, Isaac. El círculo vicioso (Runaround - 1942). Inteligencia Educativa. Instituto de Formación Tecnológica. [fecha de consulta 4 febrero 2020]. Disponible en: http://inteligenciaeducativa.net/descargas/runaround.pdf p. 3 ${ }^{37}$ Ibidem, p. 13.

${ }^{38}$ Ibidem, p. 13 y 18.
} 
Esta configuración se puede resumir en que existe para un robot una relación entre los términos de supervivencia propia (Regla 3), ordenanza humana (Regla 2) y bien humano (Regla 1) y que necesariamente se encuentran en ese orden jerárquico de menor a mayor importancia.

Para Asimov, estos son los tres niveles de valoración que debe seguir todo ente robótico o imbuido con inteligencia artificial. Lo que consigna a entender estas tres normas técnicas como axiológicas en un sentido amplio, puesto que presentan una decisión entre un actuar considerado acorde -dígase bueno- a las leyes o contrario a éstas -es decir malo-.

De tal modo, las tres leyes sirven como parámetro de lo que puede o no puede hacer un robot de forma práctica lo que de manera drástica implicaría desde la primera regla, la ausencia de daño intencionado u ordenado a otro ser humano. Sin obedecer a dilemas morales como presenta muchas veces la ética, ante situaciones de doble efecto por considerar un ejemplo. Igualmente, sirven de parámetro sobre los tipos de configuración que debe tener un sistema a nivel programación ya que esto definiría la amplitud o especificidad de la tarea a realizar.

Ahora, si a esta practicidad agregamos la característica de contextualidad y de progresividad, podemos bosquejar algunas ideas de cómo la aplicabilidad a casos concretos de lo dicho por Asimov asemeja más a toda una experiencia -actual o hipotética- donde el derecho tiene una injerencia.

Regresemos para ello a uno de los ejemplos puestos en este recorrido. Primeramente las LAWS son como ya se dijo una realidad a la que el Derecho no le ha puesto la suficiente atención. Éstas, si las vemos respecto a Las Leyes de Asimov, son completamente opuestas a la primera y segunda regla dado que el propósito de esta tecnología es justo hacer un daño a una persona humana por orden expresa de otro ser humano. Viéndolo desde la perspectiva y experiencia de este autor de Sci-Fi, entonces una obligación del Derecho tendría que ser la total prohibición de este tipo de aplicaciones ante la contradicción que representa a la robótica, incluso esta prohibición tendría que darse desde su desarrollo puesto que es en la programación donde se daría la inconsistencia.

Esta inconsistencia se da en la propia concepción de Las Leyes ante el funcionamiento progresivo de la máquina. Para que un robot pueda cumplir cabalmente el propósito de las LAWS la primera ley debe verse a la inversa de cómo fue planteada "un robot puede hacer daño a un ser humano" sin embargo, no es un interés que cualquier ser humano pueda ser dañado sino solo un grupo específico de individuos, entonces la ley debe expresarse de esta forma "un robot no puede hacer daño a algunos seres humanos" ya que desde la lógica 
computacional plantearlo como "un robot puede hacer daño a algunos seres humanos" deja la posibilidad de dañar o no al grupo lo que sería un factor para que el LAWS no realice su misión. En cambio, la negación de un grupo a ser atacado excluye la variable de posibilidad diferenciando a aquellos que sí deben de aquellos que no deben ser atacados, es decir, la máquina tendría un criterio de discriminación entre unos y otros obligando a que sea mucha mayor su certeza para no ir en contra de su protocolo.

$\mathrm{Al}$ mismo tiempo, ninguna de estas formas de redacción es suficiente para cumplir el imperativo expuesto por Asimov, por lo que para lograr el cometido de que las $L A W S$ cumpla con la regla el cambio no debe ser en la redacción de la norma sino en su fondo, específicamente en el significado de ser humano.

Si generalizamos la idea de persona humana a cualquier ente que cumpla con las características homo sapiens entonces el objetivo de las LAWS no podría cumplirse, sin embargo, si le quitamos la característica de ser humano a un grupo de individuos entonces sí, la regla puede ser cumplida puesto que ese grupo para la máquina ya no sería parte de la prohibición sino otra cosa -por usar la terminología popular ante el fenómeno de la guerra-, los monstruos.

Si vemos desde el Derecho esta experiencia gramatical descrita en el contexto de las $L A W S$ vemos entonces ciertas equivalencias con algunos principios fundamentales. En primer lugar, si vemos desde el principio de seguridad jurídica las cuatro posibles estructuras, vemos entonces que la primeramente planteada y la tercera por el calificativo de ser posibilidades abiertas, generarían una incertidumbre o laguna sobre cuándo es aplicable o no el daño a la persona o al grupo y peor aún si a esto agregamos la posibilidad de la orden dada por otro ser humano, ya que sería no nada más la ambigüedad de aplicación, sino también la ambigüedad de -principio de- legitimidad de una persona para dar la orden.

Este galimatías del principio de seguridad jurídica no es comparable al que se genera si se evalúan los $L A W S$ y a Asimov ante el principio de Igualdad o de No Discriminación. Para este principio la primera de las aseveraciones es una incierta igualdad entre los posibles afectados, ya que no existe una discriminación y dependería de la vista del humano que ordena y la normatividad secundaria definir quien merecería ser o no dañado por la máquina o por la orden humana. Esto no es así con las otras tres aseveraciones pues la segunda, por su parte, propone una inequidad clara entre quienes sí pueden ser violentados y quienes no -y que si se contextualiza a la aplicación práctica de los LAWS-, esto significaría dañar a los enemigos y no dañar a los compañeros. En este sentido la tercera de las exposiciones no es tan tajante puesto que deja 
abierta la posibilidad para una decisión de si dañar o no al contrario, pero que generaría una ambivalencia entre las leyes puesto que dependería de los protocolos de discriminación o de la orden humana un posible resultado. Finalmente y quizá el más contrario al principio sería el cuarto de los enunciados, puesto que significaría eliminar la igualdad entre las personas por medio del derecho a la dignidad humana de aquellos considerados enemigos para que puedan estar fuera de la Primera Ley, e incluso poder ordenar con justificación su daño cuando atendemos a la realidad de la segunda Ley.

En resumen, pasar por el crisol de las tres Leyes de la Robótica la aplicación de IA en las Lethal Autonomous Weapon Systems muestra que no existe forma alguna en la que sean compatibles, por lo que si el Derecho utilizara como fuente este emblema de la Ciencia Ficción para evaluar la juridicidad de la experiencia actual que representan las $L A W S$, poco quedaría en duda que éstas van en contra de los principios más esenciales del Derecho y que, por tanto, su limitación y/o regulación es una tarea no pendiente, sino urgente a corregir por la Ciencia Jurídica.

Así pues, hablando desde la corriente iusfilosófica expuesta, la herramienta que puede representar al Derecho de la Ciencia Ficción ante una experiencia como el avance tecnológico, específicamente de la Inteligencia Artificial, no solo permite al jurista hallar una solución al caso concreto sino que muestra una faceta de la transformación que está sufriendo su profesión y que si no es capaz de limitar, incentivar, regir o corregir por su propio espíritu, será esta experiencia jurídica la que limite, incentive, rija o corrija lo que significa Derecho en el devenir histórico.

\section{Bibliografía}

Asamblea General de las Naciones Unidas. Declaración Universal de los Derechos Humanos. 217 A (III) Francia, 10 diciembre 1948 [fecha de consulta 24 enero 2020]. Disponible en: https://www.un.org/es/universal-declaration-human-rights/

Asimov, Isaac. Asimov on Science Fiction. EUA, Harper Collins Publishers, 1983, ISBN 978-02-4612-0441

-----, El círculo vicioso (Runaround - 1942). Inteligencia Educativa. Instituto de Formación Tecnológica. [fecha de consulta 4 febrero 2020]. Disponible en http:// inteligenciaeducativa.net/descargas/runaround.pdf.

Borge, Iván Mateo. "La robótica y la inteligencia artificial en la prestación de servicios jurídicos” En Inteligencia Artificial, Tecnología y Derecho. España, Tirant Lo Blanch, 2017, ISBN 978-84-9169-720-6, p. 123-150 
Buchanan, Bruce G. Et Headrick, Thomas E. "Some speculation about artificial intelligence and legal reasoning”, Stanford Law Review, Volumen 23, No. 1, año 1970, noviembre, p. 40-62

Buchanan, Bruce G. "A (Very) brief history of Artificial Intelligence”, AI Magazine, American Association for Artificial Intelligence (AAAI), Volumen 26, No. 4, año 2006, invierno 2005, p. 53-60

Casanovas, Pompeu “Inteligencia Artificial y Derecho: a vuelapluma”, Teoría y derecho: revista de pensamiento jurídico. No. 7, año 2010, p. 203-222. Versión del autor disponible en Researchgate.net

Granfield, David. La experiencia interna del derecho. Una jurisprudencia de la subjetividad. México, Catholic University of America/ Universidad Iberoamericana, 1996, ISBN 968-859-228-5

Jordan, Philipp; Mubin, Omar; Obaid, Mohammad \& Sila, Paula A. "Exploring the referral and Usage of Science-Fiction in HCI Literature”. En Marcus, Aaron \& Wang, Wentao (Eds.) Design, user experience, and usability: Designing Intearctions, Tomo II, EUA, Springer, ISBN 978-3-319-91802-0, p. 19-38 Versión del autor disponible en Researchgate.net

Krausova, Alzbeta. "Intersections between Law and Artificial Intelligence” International Journal of Computer (IJC), Volumen 27, No. 1, año 2017, p. 55-68. Versión del autor disponible en Researchgate.net

Llano Alonso, Fernando H., El formalismo jurídico y la Teoría Experiencial del Derecho. España. Tirant Lo Blanch, 2009, ISBN 978-84-9985-511-0

OECD, “AI Conference Welcome Message” [en línea]. En Going Digital. AI Intelligent machines, Smart policies. 26 octubre 2017 [fecha de consulta 20 diciembre 2019]. Disponible en: https://www.oecd.org/going-digital/ ai-intelligent-machines-smart-policies/conference-agenda/

Vigo, Rodolfo L. "Los principios jurídicos y su impacto en la teoría actual." En Vigo, Rodolfo L. La interpretación (argumentación) jurídica en el Estado de Derecho Constitucional. México, Tirant Lo Blanch, 2017, ISBN 978-84-9169-616-2

Vigo, Rodolfo L. Interpretación Jurídica: del modelo iuspositivista legalista decimonónico a las nuevas perspectivas. Argentina. Rubinzal. 1999, ISBN 978-950-727-197-7 